\title{
Differentiation Potential of Mesenchymal Stem Cells Is Related to Their Intrinsic Mechanical Properties
}

\author{
Ihn Han ${ }^{1}$, Byung-Su Kwon ${ }^{2,3}$, Hun-Kuk Park ${ }^{4,5,6}$, Kyung Sook Kim ${ }^{4,5}$ \\ ${ }^{1}$ Plasma Bioscience Research Center, Kwangwoon University, Seoul, Korea \\ ${ }^{2}$ Department of Obstetrics and Gynecology, Pusan National University School of Medicine, Busan, Korea \\ ${ }^{3}$ Biomedical Research Institute and Pusan Cancer Center, Pusan National University Hospital, Busan, Korea \\ ${ }^{4}$ Department of Biomedical Engineering, Graduate School, Kyung Hee University, Seoul Korea \\ ${ }^{5}$ Program of Medical Engineering, Kyung Hee University, Seoul, Korea \\ ${ }^{6}$ Healthcare Industry Research Institute, Kyung Hee University, Seoul, Korea
}

Purpose: The differentiation properties of stem cells are not yet fully understood due to their close association with multiple environmental and extrinsic factors. This study investigates the differentiation properties of mesenchymal stem cells (MSCs) and correlates them with their intrinsic mechanical properties.

Methods: A total of 3 different types of MSCs, namely bone marrow-derived MSCs (BMSCs), umbilical cord-derived MSCs (UCSCs), and adipose-derived MSCs (ADSCs) were evaluated. These 3 MSCs were individually differentiated into adipocytes and osteoblasts for 3 weeks. The mechanical properties of the MSCs and differentiated cells were determined by atomic force microscopy.

Results: ADSCs showed the greatest ability to differentiate into adipocytes, followed by BMSCs and UCSCs. While UCSCs differentiated readily into osteoblasts, BMSCs and ADSCs were less likely to undergo this differentiation. UCSCs were the "hardest" cells, while ADSCs were the "softest." The cells differentiated from "hard" MSCs were stiffer than the cells differentiated from "soft" MSCs, irrespective of lineage specification.

Conclusions: The differentiation ability of MSCs and the mechanical properties of the differentiated cells were closely linked. However, there were no significant correlations regarding changes in the mechanical properties between the nuclear region and the cytoplasm during differentiation.

Keywords: Mechanics; Mesenchymal stem cells; Adipogenesis; Osteogenesis; Atomic Force Microscopy

- Research Ethics: This study was approved by the Institutional Review Board (IRB) of Kyung Hee Medical Center (approval number: KMC IRB 1423-01).

- Conflict of Interest: No potential conflict of interest relevant to this article was reported.

\section{- HIGHLIGHTS}

- UCSCs were the "hardest" cells, while ADSCs were the "softest."

- Hard and soft MSCs well differentiated into osteoblast and adipocytes, respectively.

- Cells differentiated from hard MSCs were stiffer than that from soft MSCs.

\section{INTRODUCTION}

Mesenchymal stem cells (MSCs) have been considered a prom- ising stem cell source for the regeneration of damaged cells and tissues because they meet the requirement of stem cells, including being in an undifferentiated state and having the capacity to
Corresponding author: Kyung Sook Kim (iD http://orcid.org/0000-0003-3572-1169 Department of Biomedical Engineering, College of Medicine, Kyung Hee University, 26 Kyungheedae-ro, Dongdaemun-gu, Seoul 02447, Korea E-mail: moosou94@khu.ac.kr / Tel: +82-2-961-9501 / Fax: +82-2-6008-5535 Submitted: March 23, 2017 / Accepted after revision: April 6, 2017
This is an Open Access article distributed under the terms of the Creative Commons Attribution Non-Commercial License (http://creativecommons.org/licenses/by-nc/4.0/) which permits unrestricted non-commercial use, distribution, and reproduction in any medium, provided the original work is properly cited. 
differentiate into various lineages, in addition to having the ability to self-renew. Also, MSCs are not subject to ethical issues in acquisition, use or propagation [1-4]. MSCs can be easily isolated from a variety of tissues in the human body, such as bone marrow, umbilical cord, and adipose tissue, among others [5-7].

Bone marrow-derived MSCs (BMSCs) have been used mainly for tissue regeneration; however, the utility of these cells is limited due to the highly invasive isolation procedure for bone marrow [5,8]. Umbilical cord-derived MSCs (UCSCs) are a good alternative to BMSCs because they can be obtained by a less invasive method, they are similar to BMSCs with respect to their capacity as stem cells, and they are less aged than other MSCs [9-11]. Recently, adipose-derived MSCs (ADSCs) have become popular because they can be isolated in large amounts by the least invasive method of all the stem cells [12-14].

MSCs, including BMSCs, UCSCs, ADSCs, have the common stem cell characteristics mentioned above; however, other properties, including colony formation, proliferative capacity, and the ability to differentiate depend on the MSC type. Several studies have reported a comparative analysis of these MSCs $[5,8,15]$. In these studies, the UCSCs exhibited the highest proliferation capacity with the longest culture period and lowest colony-formation ability, BMSCs had the lowest proliferation capacity and a short culture period $[5,15]$. ADSCs demonstrated the highest colony formation ability [5]. ADSCs and BMSCs differentiated well into adipocytes, but UCSCs differentiated poorly [16]. However, in other reports by Shafiee et al. [8] and Zhang et al. [15], BMSCs showed the strongest ability to undergo osteogenic differentiation, followed by UCSCs and ADSCs $[8,15]$. In a report by Kern et al. [5], UCSCs were able to more actively differentiate to osteoblasts than BMSCs and ADSCs.

The differentiation tendencies of stem cells are closely linked to several factors including adhesive contexts, mechanical signals, and the physical responses of the cells [17]. Recently, the mechanical properties of the extracellular matrix (ECM) of MSCs have become an area of interest because the elastic properties of the ECM significantly affect differentiation. Stem cells on a soft ECM or matrix $(\mathrm{E}=0.1-1 \mathrm{kPa}$, where $\mathrm{E}$ denotes the elasticity of the matrix) are more likely to differentiate into neurons, whereas cells on a hard matrix $(\mathrm{E}=25-40 \mathrm{kPa})$ are more likely to differentiate into osteoblasts [18]. Differentiation is also closely linked to the intrinsic mechanical properties, including the elasticity and viscosity of individual MSCs. González-Cruz et al. [19] reported that, among ADSCs from the same source that were treated under the same conditions, the stiffest cells tended to differentiate into osteoblasts while the "softest" cells tended to differentiate into adipocytes.

In this study, the changes of physical stiffness were compared among 3 different MSCs, including UCSCs, BMSCs, and ADSCs, throughout the induction period of differentiation for a better understanding of the correlation among their properties including the source, the differentiation potential, and the physical stiffness. The changes of physical stiffness were also compared between nuclear and cytoplasmic regions of bipotent mesenchymal progenitor cells throughout the induction period of differentiation.

\section{MATERIALS AND METHODS}

\section{Isolation of ADSCs and UCSCs}

Human umbilical cord tissue was obtained from full-term infants born by Caesarian section to 14 women (mean age, 28.3 years) who had no complications throughout pregnancy, conducted under the approval of the Institutional Review Board (IRB) of Kyung Hee Medical Center (approval number: KMC IRB 1423-01). Written informed consent was obtained from all participants prior to using umbilical cord tissue. Isolated umbilical cord tissues were cut into $1-2 \mathrm{~mm}^{3}$ pieces and enzymatically digested for $3-4$ hours at $37^{\circ} \mathrm{C}$ with $3 \mathrm{mg} / \mathrm{mL}$ type I collagenase. Adipose tissue was obtained from full-term maternal fat from the same women, immersed in Hank's balanced salt solution, and immediately transferred to the laboratory. ADSCs and UCSCs were cultured according to the established methods in growth media from the EGM-2 kit, and the medium was changed every 2-3 days [20].

\section{BMSC Culture}

BMSCs were purchased from the American Type Culture Collection (Manassas, VA, USA) and were cultured in the same manner as the ADSCs and UCSCs, as described above.

\section{Flow Cytometry Analysis}

For cell surface antigen phenotyping, 5- to 7-passage cells were analyzed by FACS Calibur (BD Bioscience, San Jose, CA, USA). Cells were detached using BD Accutase cell detachment solution (Cat. No. 561527, BD Bioscience), harvested, and washed twice with BD Pharmingen Stain Buffer (Cat. No. 554656, BD Bioscience). The assay procedure described in the human MSC analysis kit (Cat. No. 562245, BD Bioscience) was then used. 


\section{Differentiation into Adipocytes and Osteoblasts}

All MSCs were individually differentiated in adipogenic (Cat No. GUXMX-90031, Cyagen Biosciences, Santa Clara, CA, USA) and osteogenic differentiation medium (Cat No. GUXMX-90021, Cyagen Biosciences). The cells in the adipogenic and osteogenic differentiation medium were then stained with oil red O (Sigma-Aldrich, St. Louis, MO, USA) and von Kossa (Sigma-Aldrich) for 10 minutes. For the quantification of mineralization by measuring calcium nodules, the differentiated cells were stained with alizarin red $\mathrm{S}$ and resolved with acetic acid. The supernatant was analyzed by measuring the absorbance at $490 \mathrm{~nm}$ using a spectrophotometer. The differentiation steps used for both adipocytes and osteoblasts were determined from established protocols $[6,17]$.

\section{Atomic Force Microscopy}

The mechanical property of stiffness of the MSCs was investigated using atomic force microscopy (AFM) with the NANO Station II (Surface Imaging Systems, Herzogenrath, Germany).
Force-distance (FD) curves were measured in contact mode using a gold-coated probe in a solution at about 15 times per cell ( $>20$ cells were measured per group).

\section{Statistics}

The significance of the obtained results was assessed by 2-way analysis of variance (SPSS ver. 13, SPSS Inc., Chicago IL, USA). P-values less than 0.05 were considered to be statistically significant.

\section{RESULTS}

\section{Characterization of MSCs}

To characterize the MSCs, the cell surface antigen phenotypes were determined by flow cytometry (FACS) for the MSCs derived from bone marrow, umbilical cord, and fat tissue. More than $95 \%$ of the 3 MSCs types expressed the typical MSCs marker proteins CD73 (surface enzyme) and CD90 (ECM protein). More than $95 \%$ of BMSCs and ADSCs expressed the typi-
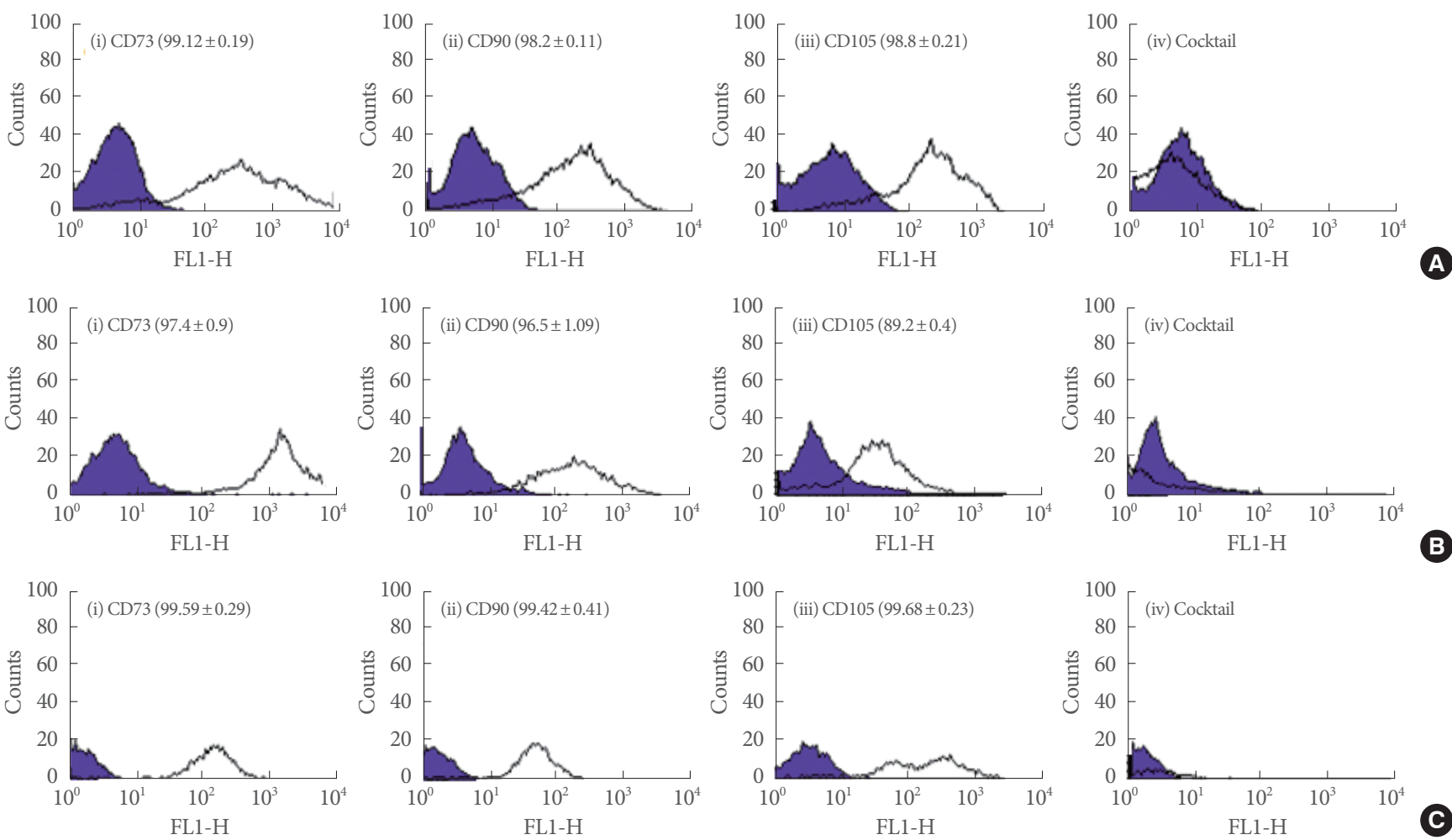

Fig. 1. (A) Phenotypic analysis of bone marrow-derived MSCs, (B) umbilical cord-derived MSCs, and (C) adipose-derived MSCs byflow cytometry. MSCs-associated markers (CD73, CD90, and CD105) and hematopoietic lineage markers (CD34, CD45, CD11b or CD14, CD19 or CD79 $\alpha$, and HLA-DR) were evaluated. The numbers shown represent the percentage of positive cells expressing each respective marker. MSCs, mescenchymal stem cells. 
cal MSCs marker protein CD105 (an ECM protein); however, the intensity of CD105 expression in UCSCs was significantly lower than that of the others. This result is consistent with the reported results. All 3 cell types were negative for expression of hematopoietic lineage markers including CD34, CD45, CD11b or CD14, CD19 or CD79 $\alpha$, and HLA-DR (Fig. 1).

All MSCs displayed spindle-shaped and fibroblast-like morphologies. BMSCs and UCSCs were morphologically homogeneous, while ADSCs were heterogeneous in shape, varying from round to cuboidal or elongated in shape. The morphology changed during differentiation, depending on the type of MSCs and the differentiation lineage. When cells differentiated into

BMSCs
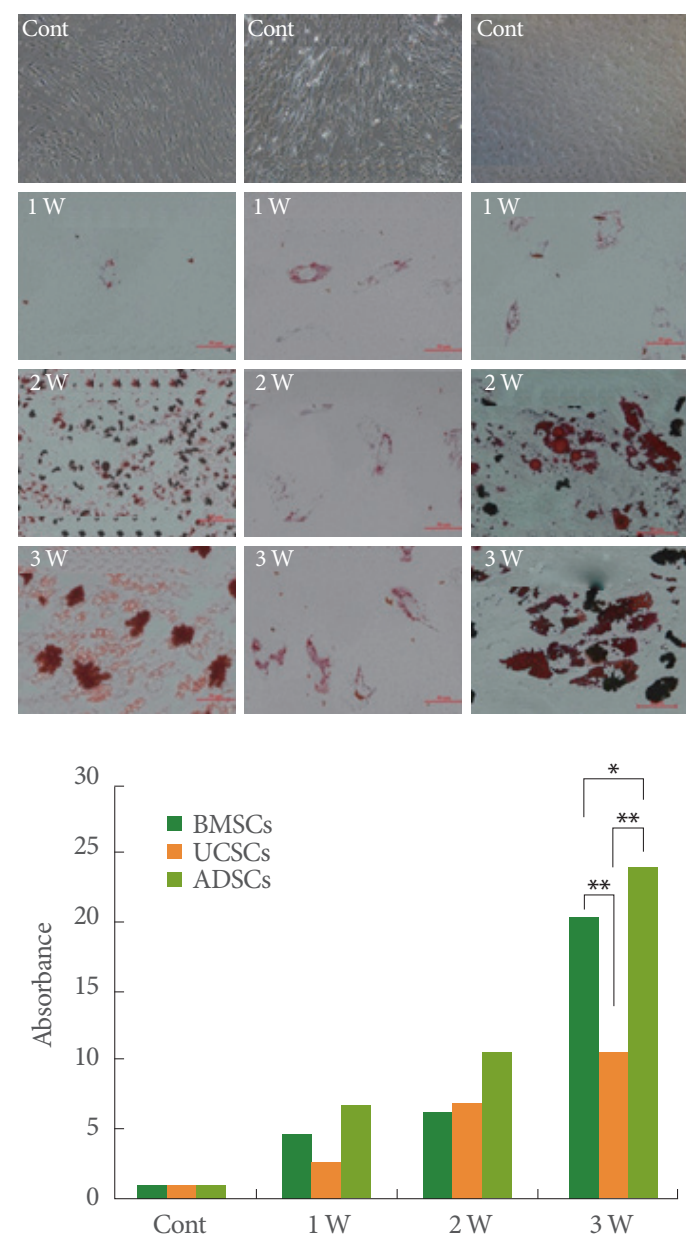

adipocytes, the BMSC-derived cells elongated and the size of the UCSC-derived cells increased (Supplementary Fig. 1A). By contrast, there were no significant changes in ADSC-derived cells. When cells differentiated into osteoblasts, the cells derived from BMSCs aggregated easily and formed a monolayer after 2 weeks of induction (Supplementary Fig. 1B), while no morphological changes were observed during the induction process for either the UCSCs or ADSCs.

\section{Multilineage Differentiation Capability of the MSCs Types}

Each of the 3 types of MSCs were differentiated into adipocytes and osteoblasts and stained every week during the induction pro-
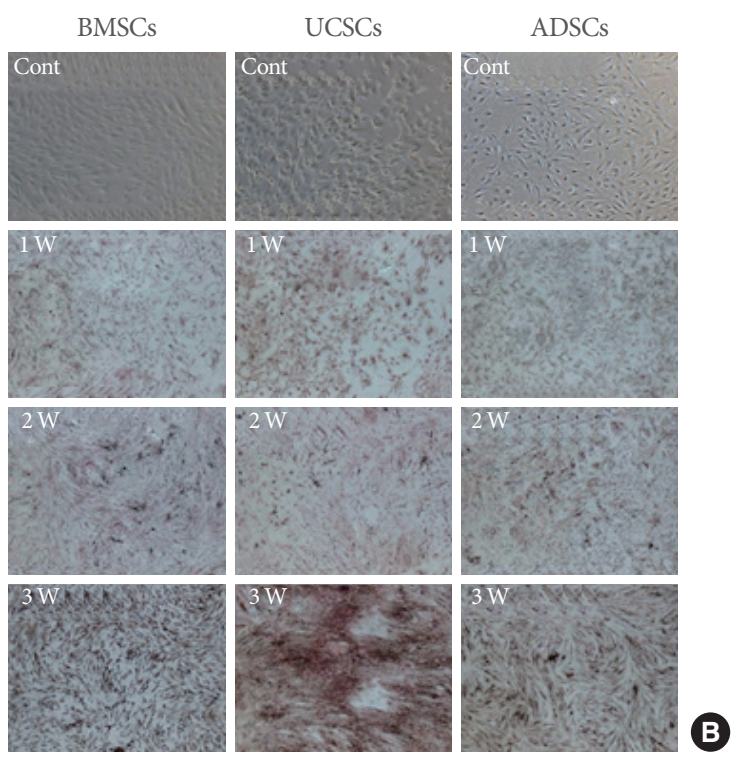

B

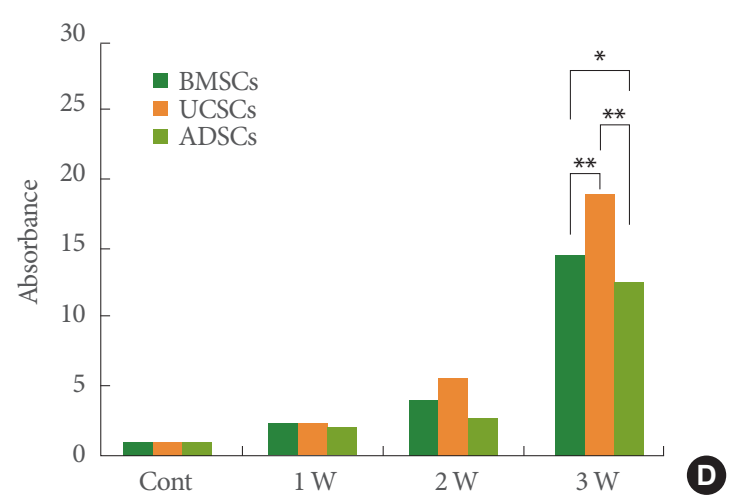

Fig. 2. (A) During adipogenic differentiation, cells were stained using oil-red O (ORO) each week from weeks 1 to 3 ( $\times 400)$. (B) Cells undergoing osteogenic differentiation were stained with von Kossa (×200). (C) Ability to differentiate into adipocytes and (D) osteoblasts was analyzed each week during induction by absorbance measurement at $520 \mathrm{~nm}$ for ORO and $490 \mathrm{~nm}$ for alizarin red S. MSCs, mescenchymal stem cells; BMSCs, bone marrow-derived MSCs; UCSCs, umbilical cord-derived MSCs; ADSCs, adipose-derived MSCs; Cont, control; 1 W, 1 week; 2 W, 2 weeks; 3 W, 3 weeks. Scale bar represents 50 m. Error bars indicate the mean \pm standard deviation. ${ }^{*} \mathrm{P}<0.05,{ }^{* *} \mathrm{P}<0.005,2$-way analysis of variance. 

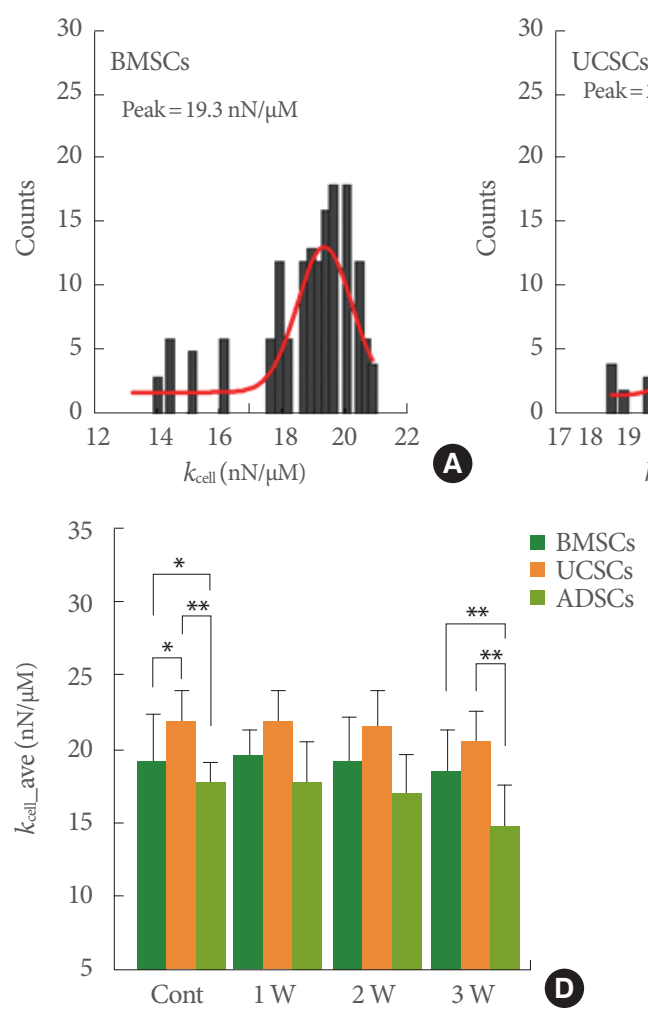
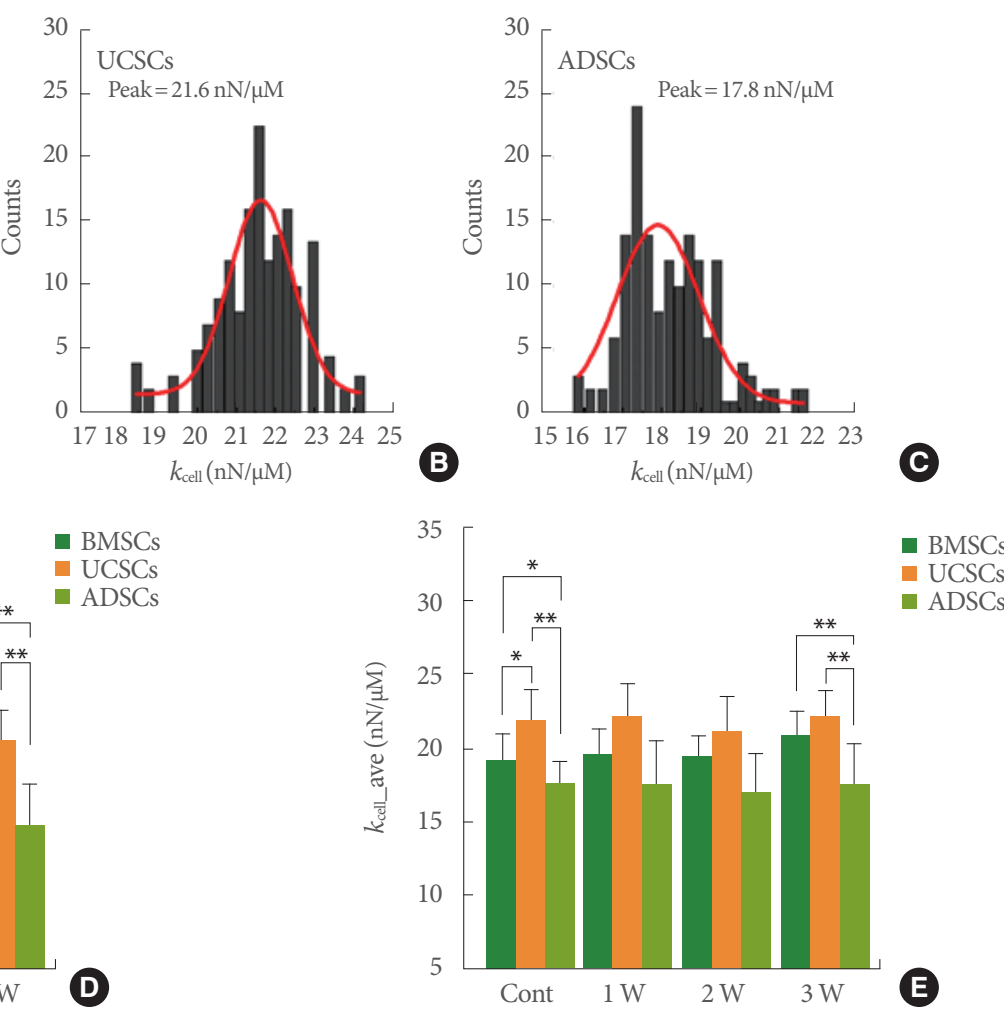

Fig. 3. (A) Stiffness distribution of BMSCs, (B) UCSCs, and (C) ADSCs, respectively. The red lines indicate the Gaussian fit of the data. All MSCs were heterogeneous in their mechanical property of stiffness, as indicated by the broad distribution of $k_{\text {cell. }}$. Averaged stiffness values $\left(k_{\text {cell_ave }}\right)$ during adipogenic (D) and osteogenic (E) induction. Green color bars indicate the result for BMSCs, and orange and yellow-green color bars correspond to the results for UCSCs and ADSCs. MSCs, mescenchymal stem cells; BMSCs, bone marrow-derived MSCs; UCSCs, umbilical cord-derived MSCs; ADSCs, adipose-derived MSCs; Cont, control; 1 W, 1 week; 2 W, 2 weeks; $3 \mathrm{~W}, 3$ weeks. Error bars indicate the mean \pm standard deviation. ${ }^{*} \mathrm{P}<0.05,{ }^{* *} \mathrm{P}<0.005,2$-way analysis of variance.

cess to provide a comparison of the differentiation potential of each. Fig. 2A and B shows the results of oil-red $\mathrm{O}$ and von Kossa staining for adipogenic and osteogenic induction, respectively. In both BMSCs and ADSCs, lipid vacuoles formed after 2 weeks, and the number of vacuoles increased as the induction time increased (Fig. 2A). The size of the lipid vacuoles also increased as differentiation progressed. On the other hand, only small lipid vacuoles were observed after 3 weeks of induction during the differentiation of UCSCs. Fluorescence-based lipid vacuole levels confirmed that BMSCs and ADSCs had higher differentiation ability than UCSCs throughout differentiation (Fig. 2C). In the case of osteogenic differentiation, a mineralized matrix was clearly observed in all MSCs after 2 weeks of induction. UCSCs showed a significantly higher osteogenic differentiation capacity than either BMSCs or ADSCs, as indicated by the high absorbance levels detected, as shown in Fig. 2D. No significant difference in mineralization was detected between BMSCs and ADSCs.

\section{Changes in Mechanical Properties During Differentiation}

The mechanical properties of the MSCs were evaluated with FD curve measurements using AFM. All 3 types of the MSCs were heterogeneous in stiffness, as defined by the broad distribution of the stiffness curve ( $\left.k_{\text {cell }}\right)($ Fig. 3A-C). The UCSCs were the "stiffest" cells, followed by BMSCs and ADSCs. During differentiation, changes in $k_{\text {cell }}$ were analyzed in several cells for

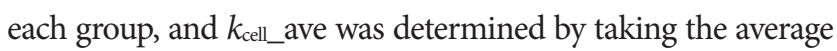

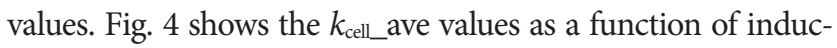
tion time. When the cells differentiated into adipocytes, all cells "softened" as induction progressed (Fig. 3D). In BMSCs and

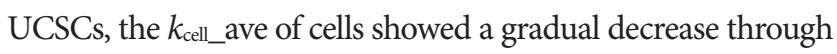
the entire differentiation process, except at 1 week of induction

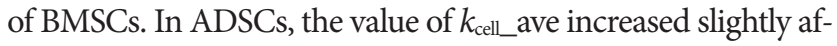
ter 1 week of induction and then rapidly dropped off after 3 weeks. At 3 weeks of induction, all adipocyte cells had smaller

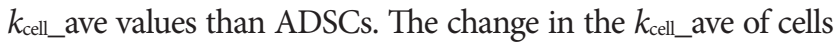



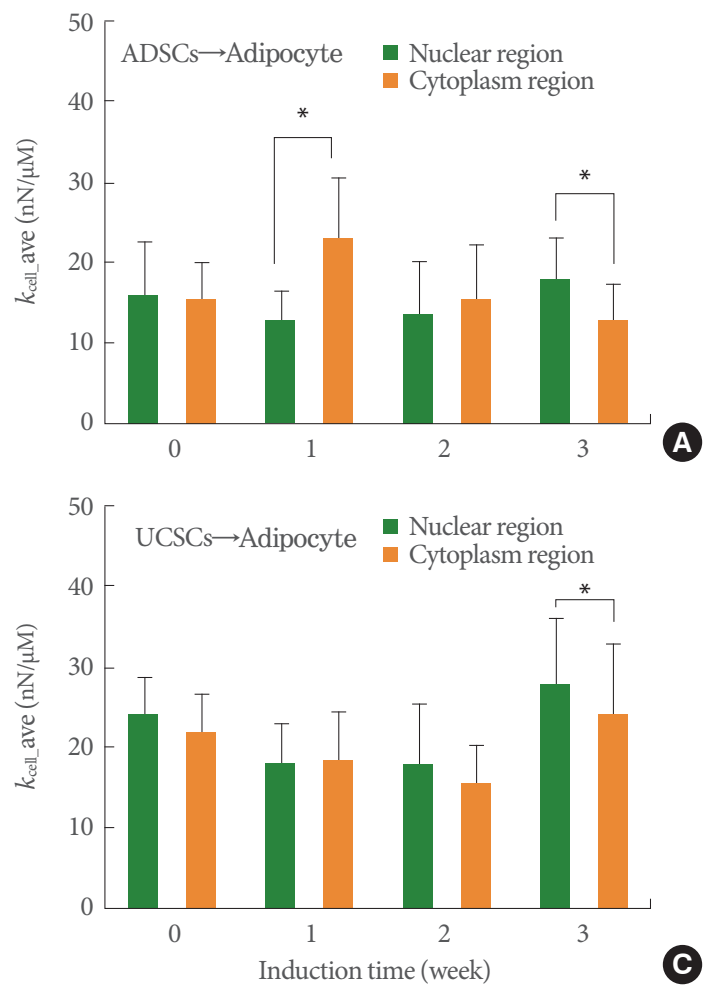
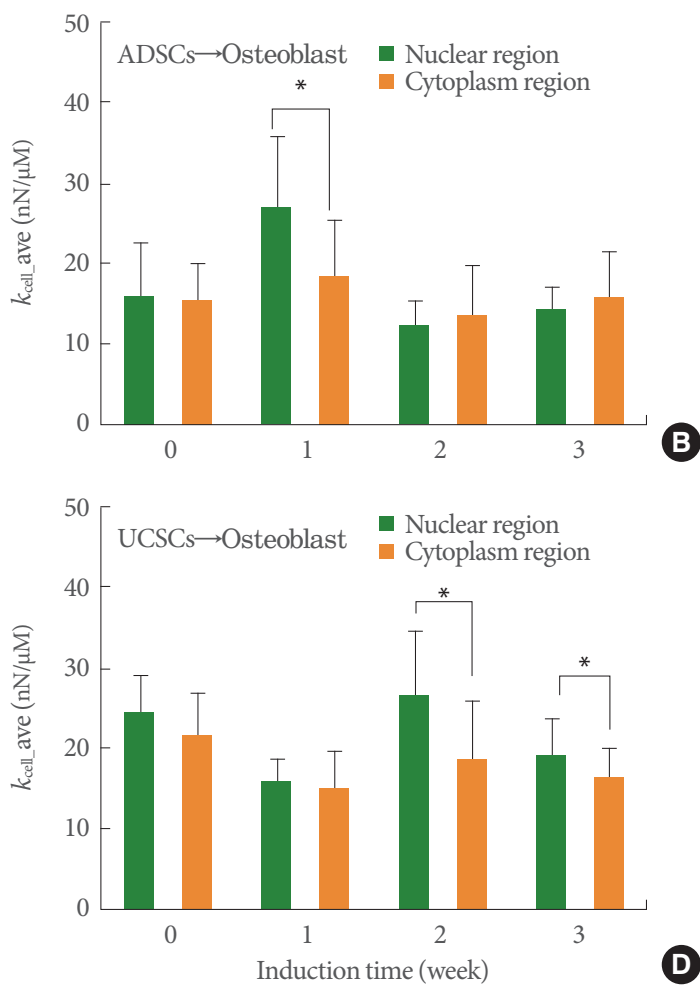

Fig. 4. Averaged stiffness values of ADSCs (A, B) and UCSCs (C, D) differentiated into adipocytes and osteoblasts, respectively. Green and orange color bars indicate the results for the nuclear regions and the cytoplasm, respectively. MSCs, mescenchymal stem cells; ADSCs, adipose-derived MSCs; UCSCs, umbilical cord-derived MSCs. ${ }^{*} \mathrm{P}<0.05$ for stiffness of the nuclear region compared with that of the cytoplasm. Error bars indicate the mean \pm standard deviation. ${ }^{*} \mathrm{P}<0.05,2$-way analysis of variance.

maintained in the osteogenic differentiation medium was more intricate than those of adipocytes (Fig. 3E). The $k_{\text {cell_ave values }}$ dropped off at 2 weeks of induction in cells from BMSCs and UCSCs, and then rapidly increased at 3 weeks of induction. The stiffness of the cells from ADSCs did not differ substantially during the process, except for a slight decrease at 1 week of induction. After 3 weeks of induction, the osteoblasts from BMSCs were "harder" than BMSCs, while the osteoblasts from UCSCs and ADSCs showed similar stiffness to their MSCs.

\section{Independent Mechanical Properties of the Nucleus and Cytoplasm During Differentiation}

For the "softest" cells (ADSCs) and the "hardest" cells (UCSCs), the mechanical changes were analyzed at 2-different regions of the nucleus and cytoplasm. During adipocyte differentiation of ADSCs, the stiffness of the nuclear region gradually decreased during the first week of induction and then gradually increased (Fig. 4A), whereas the cytoplasm stiffness peaked after 1 week and decreased thereafter. During osteoblast differentiation of
ADSCs, the stiffness did not change greatly in either the nuclear or the cytoplasm regions, except at 1 week of induction as the nuclear region became stiffer (Fig. 4B). In UCSCs, the nuclear region was "soft" during adipocyte differentiation (at 1 and 2 weeks), but became "hard" when the cells were fully differentiated (at 3 weeks) (Fig. 4C). The mechanical properties of cells that differentiated into osteoblasts showed no clear dependence on the induction time (Fig. 4D). There were no significant differences in cytoplasm or nuclear stiffness in either the adipocyte or osteoblast differentiation of UCSCs.

\section{DISCUSSION}

This study compared the differentiation capability of 3 types of MSCs, BMSCs, UCSCs, and ADSCs, by investigating their biological and mechanical properties. These MSCs showed lineage-specific differentiation properties, as is demonstrated in Fig. 2. Based on the results of 3 weeks after induction, ADSCs showed a greater ability to differentiate into adipocytes. The dif- 
ferentiation abilities of BMSCs and UCSCs were $84 \%$ and $45 \%$ of that of ADSCs, respectively. When the MSCs differentiated into osteoblasts, UCSCs displayed the strongest staining results, followed by BMSCs and ADSCs.

The mechanical property of stiffness was also dependent on the type of MSCs and the differentiation lineage induced. UCSCs were the "hardest" cells followed by BMSCs and ADSCs. These results indicated that the "hard" UCSCs more easily differentiated into osteoblasts, while the "soft" ADSCs easily differentiated into adipocytes. González-Cruz et al. [19] correlated the cellular mechanical properties of ADSCs with their differentiation potential. They isolated 32 ADSC clonal populations under the same conditions, and assessed their clones' mechanical properties and differentiation abilities. The ADSCs showed heterogeneity in both elastic $(0.6 \pm 0.2$ to $1.6 \pm 0.5 \mathrm{kPa})$ and viscoelastic $(0.7 \pm 0.4$ to $2.6 \pm 1.6 \mathrm{kPa})$ properties. The ADSCs were differentiated toward 3 different lineages, and it was revealed that there was a significant correlation between the mechanical and differentiation potential properties. Adipogenesis was negatively correlated with elasticity, while osteogenesis was positively correlated with elasticity. Chondrogenesis was positively correlated with both elasticity and viscosity. These results suggest that cellular mechanical properties can be used as biomarkers to predict the differentiation potential of ADSCs. Based on the work of González-Cruz et al. [19], we hypothesized that the intrinsic mechanical properties of MSCs may predict the differences in the differentiation potential of the 3 types of MSCs.

After having undergone 3 weeks of induction, the mechanical characteristics of the fully differentiated cells depended on the type of MSCs used. The differentiated adipocytes from UCSCs were the "hardest," while adipocytes derived from ADSCs were the "softest," as shown in Fig. 3. The osteoblasts from UCSCs had a similar stiffness to those of BMSCs, and both cell types were "harder" than the osteoblasts from ADSCs. That is, the differentiated cells derived from "hard" MSCs were stiffer than the cells derived from "soft" MSCs, regardless of lineage.

As external stimuli are transmitted from the cytoplasm to the nucleus through the cytoskeleton, the response to stimuli depends on the composition and structure of both the cytoplasm and the nucleus. The mechanical properties of the nucleus depend on the lamina and chromatin architecture [21-23]. According to Pajerowski et al. [23], in human embryonic stem cells, the shape or mechanical properties of nuclei readily change during differentiation, such that a differentiated cell nucleus is 6 times stiffer than a stem cell nucleus. Cytoskeletal components com- posed of actin, microtubules, and intermediate filaments in the cytoplasm play an important role in the elastic properties of cells. These components vary in amount and arrangement during stem cell differentiation, resulting in changes in elasticity [24-26].

ADSCs had a fibroblast-like shape, and most of the cells were flat. UCSCs were typically thin and long, with a spindle shape (Supplementary Fig. 2). In the AFM images, the nucleus appeared brighter than the other regions because of its height. The heights of the ADSCs and UCSCs were $5.5 \mu \mathrm{m}$ and $7.5 \mu \mathrm{m}$, respectively. The difference in thickness between the nucleus and the cytoplasm was about $1-2 \mu \mathrm{m}$. The cytoplasm over the nucleus seemed to be very thin, and the cytoskeleton was well-developed around the nucleus - not over the nucleus. It was assumed, therefore, that the cytoplasm on the nucleus probably did not have a significant influence on the stiffness of the nucleus.

The changes in the mechanical properties of the nuclear region seem to be independent of the changes in the cytoplasm. The ADSC nuclear region initially had similar stiffness to that of the cytoplasm (Supplementary Fig. 3). The stiffness of both the nuclear and cytoplasmic regions changed during differentiation, and these changes were significant at 1 week of induction for all cases. The cytoplasm was stiffer than the nuclear region when ADSCs differentiated into adipocytes, whereas the nuclear region was stiffer than the cytoplasm during osteogenic differentiation. Alongside this, the difference in stiffness between the nuclear region and the cytoplasm at 3 weeks of induction when differentiation was almost complete was in contrast to the stiffness at 1 week. However, this difference was not statistically significant for osteogenic differentiation. In the case of UCSCs, the nuclear region showed a similar stiffness to the cytoplasm in the early stages of differentiation in both lineages, but the difference in stiffness between the 2 regions increased as induction proceeded, and was statistically significant at week 3 in both lineages.

In conclusion, the differentiation ability of MSCs and the mechanical properties of the differentiated cells were closely linked. In MSCs, the changes in the mechanical properties of the nuclear region during differentiation showed no correlation with those of the cytoplasm.

\section{SUPPLEMENTARY MATERIALS}

Supplementary Figures 1-3 can be found via https://doi.org/ 10.5213/inj.1734856.428. 


\section{REFERENCES}

1. Azizi SA, Stokes D, Augelli BJ, DiGirolamo C, Prockop DJ. Engraftment and migration of human bone marrow stromal cells implanted in the brains of albino rats: similarities to astrocyte grafts. Proc Natl Acad Sci U S A 1998;95:3908-13.

2. Young RG, Butler DL, Weber W, Caplan AI, Gordon SL, Fink DJ. Use of mesenchymal stem cells in a collagen matrix for Achilles tendon repair. J Orthop Res 1998;16:406-13.

3. Alhadlaq A, Mao JJ. Mesenchymal stem cells: isolation and therapeutics. Stem Cells Dev 2004;13:436-48.

4. Ferrari G, Cusella-De Angelis G, Coletta M, Paolucci E, Stornaiuolo A, Cossu G, et al. Muscle regeneration by bone marrow-derived myogenic progenitors. Science 1998;279:1528-30.

5. Kern S, Eichler H, Stoeve J, Klüter H, Bieback K. Comparative analysis of mesenchymal stem cells from bone marrow, umbilical cord blood, or adipose tissue. Stem Cells 2006;24:1294-301.

6. Stanko P, Kaiserova K, Altanerova V, Altaner C. Comparison of human mesenchymal stem cells derived from dental pulp, bone marrow, adipose tissue, and umbilical cord tissue by gene expression. Biomed Pap Med Fac Univ Palacky Olomouc Czech Repub 2014; 158:373-7.

7. Peng L, Jia Z, Yin X, Zhang X, Liu Y, Chen P, et al. Comparative analysis of mesenchymal stem cells from bone marrow, cartilage, and adipose tissue. Stem Cells Dev 2008;17:761-73.

8. Shafiee A, Seyedjafari E, Soleimani M, Ahmadbeigi N, Dinarvand P, Ghaemi N. A comparison between osteogenic differentiation of human unrestricted somatic stem cells and mesenchymal stem cells from bone marrow and adipose tissue. Biotechnol Lett 2011; 33:1257-64.

9. Xu Y, Meng H, Li C, Hao M, Wang Y, Yu Z, et al. Umbilical cordderived mesenchymal stem cells isolated by a novel explantation technique can differentiate into functional endothelial cells and promote revascularization. Stem Cells Dev 2010;19:1511-22.

10. Fan CG, Zhang QJ, Zhou JR. Therapeutic potentials of mesenchymal stem cells derived from human umbilical cord. Stem Cell Rev 2011;7:195-207.

11. Hatlapatka T, Moretti P, Lavrentieva A, Hass R, Marquardt N, Jacobs R, et al. Optimization of culture conditions for the expansion of umbilical cord-derived mesenchymal stem or stromal cell-like cells using xeno-free culture conditions. Tissue Eng Part C Methods 2011;17:485-93.

12. Ogawa R, Mizuno H, Watanabe A, Migita M, Shimada T, Hyakusoku H. Osteogenic and chondrogenic differentiation by adiposederived stem cells harvested from GFP transgenic mice. Biochem
Biophys Res Commun 2004;313:871-7.

13. Estes BT, Diekman BO, Gimble JM, Guilak F. Isolation of adiposederived stem cells and their induction to a chondrogenic phenotype. Nat Protoc 2010;5:1294-311.

14. Estes BT, Guilak F. Three-dimensional culture systems to induce chondrogenesis of adipose-derived stem cells. Methods Mol Biol 2011;702:201-17.

15. Zhang ZY, Teoh SH, Chong MS, Schantz JT, Fisk NM, Choolani MA, et al. Superior osteogenic capacity for bone tissue engineering of fetal compared with perinatal and adult mesenchymal stem cells. Stem Cells 2009;27:126-37.

16. Rebelatto CK, Aguiar AM, Moretão MP, Senegaglia AC, Hansen P, Barchiki F, et al. Dissimilar differentiation of mesenchymal stem cells from bone marrow, umbilical cord blood, and adipose tissue. Exp Biol Med (Maywood) 2008;233:901-13.

17. Cohen DM, Chen CS, Mechanical control of stem cell differentiation. StemBook [Internet]. Cambridge (MA): Harvard Stem Cell Institute; 2008.

18. Engler AJ, Sen S, Sweeney HL, Discher DE. Matrix elasticity directs stem cell lineage specification. Cell 2006;126:677-89.

19. González-Cruz RD, Fonseca VC, Darling EM. Cellular mechanical properties reflect the differentiation potential of adipose-derived mesenchymal stem cells. Proc Natl Acad Sci U S A 2012;109: E1523-9.

20. Carvalho JL, Braga VB, Melo MB, Campos AC, Oliveira MS, Gomes DA, et al. Priming mesenchymal stem cells boosts stem cell therapy to treat myocardial infarction. J Cell Mol Med 2013;17:61725.

21. Chalut KJ, Kulangara K, Giacomelli MG, Wax A, Leong KW. Deformation of stem cell nuclei by nanotopographical cues. Soft Matter 2010;6:1675-81.

22. Dahl KN, Ribeiro AJ, Lammerding J. Nuclear shape, mechanics, and mechanotransduction. Circ Res 2008;102:1307-18.

23. Pajerowski JD, Dahl KN, Zhong FL, Sammak PJ, Discher DE. Physical plasticity of the nucleus in stem cell differentiation. Proc Natl Acad Sci U S A 2007;104:15619-24.

24. Chen Q, Xiao P, Chen JN, Cai JY, Cai XF, Ding H, et al. AFM studies of cellular mechanics during osteogenic differentiation of human amniotic fluid-derived stem cells. Anal Sci 2010;26:1033-7.

25. Yourek G, Hussain MA, Mao JJ. Cytoskeletal changes of mesenchymal stem cells during differentiation. ASAIO J 2007;53:219-28.

26. Rodríguez JP, González M, Ríos S, Cambiazo V. Cytoskeletal organization of human mesenchymal stem cells (MSC) changes during their osteogenic differentiation. J Cell Biochem 2004;93:721-31. 

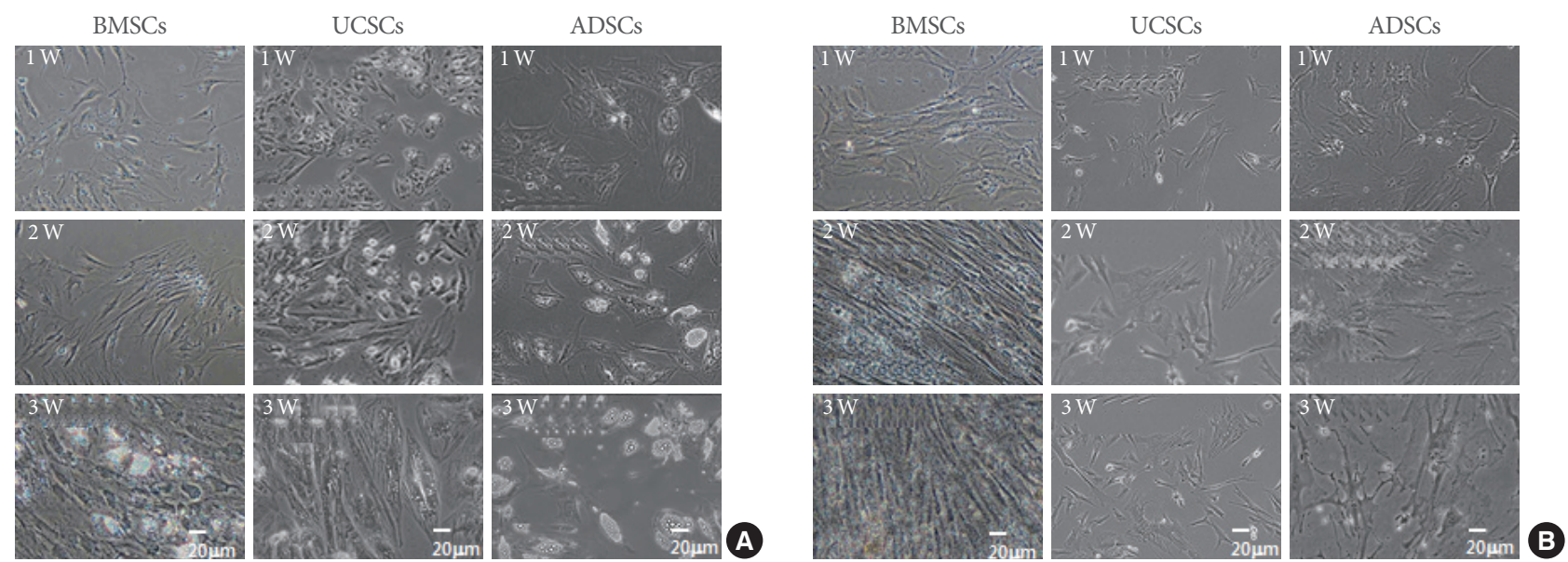

Supplementary Fig. 1. Light microscopy images of BMSCs, UCSCs, and ADSCs under adipogenic (A) and the osteogenic (B) induction conditions $(\times 400)$. The images were taken from weeks 1 to 3 after induction. MSCs, mescenchymal stem cells; BMSCs, bone marrow-derived MSCs; UCSCs, umbilical cord-derived MSCs; ADSCs, adipose-derived MSCs; 1 W, 1 week; 2 W, 2 weeks; 3 W, 3 weeks. Scale bar represents $20 \mu \mathrm{m}$. 

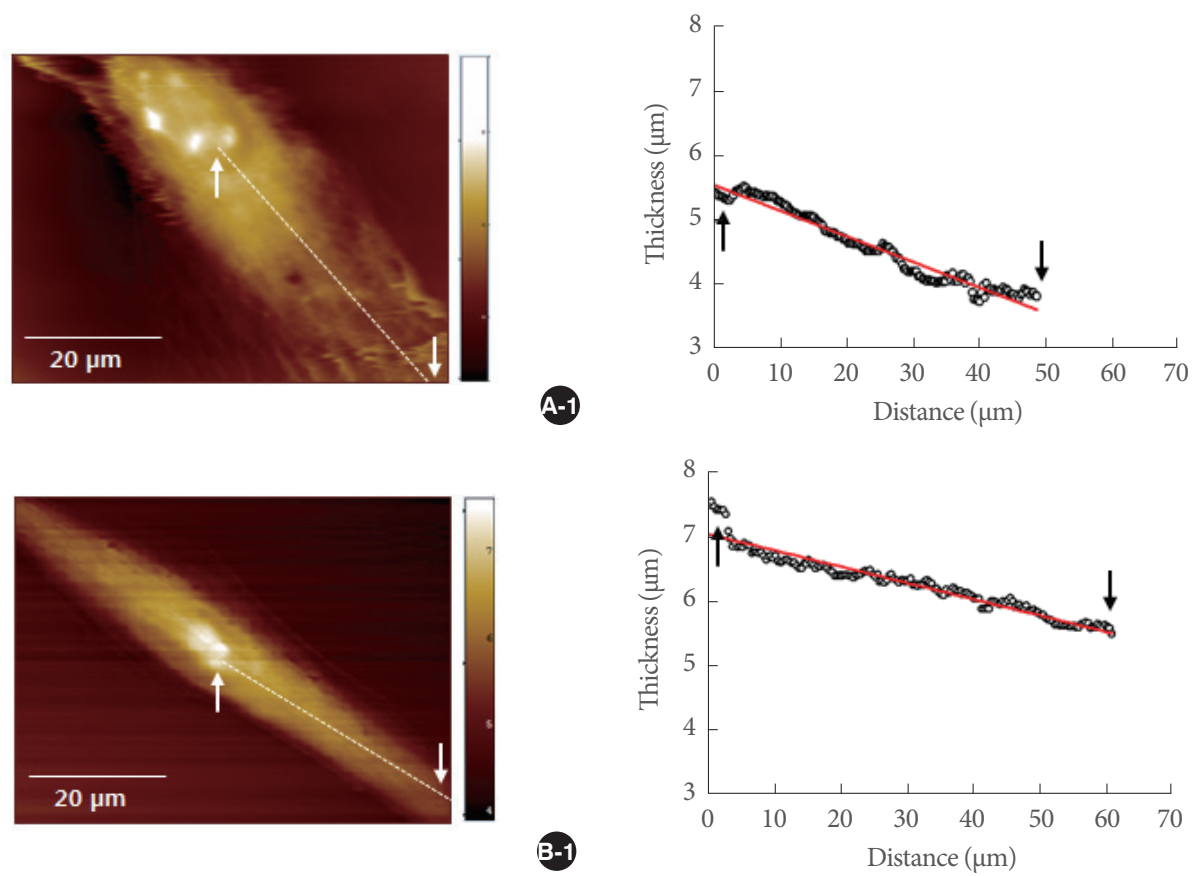

Supplementary Fig. 2. Atomic force microscopy (AFM) images of ADSCs (A-1) and UCSCs (B-1), respectively. The different colors in the images indicate different heights of the cells: the light and dark colors correspond to higher and lower regions, respectively. The line profiles taken from AFM images are indicated by white dotted lines (A-2, B-2). The start and end points are indicated by white (A-1, B-1) and dark arrows (A-2, B-2). The red lines indicate the linear fit to the data. MSCs, mescenchymal stem cells; ADSCs, adipose-derived MSCs; UCSCs, umbilical cord-derived MSCs. 

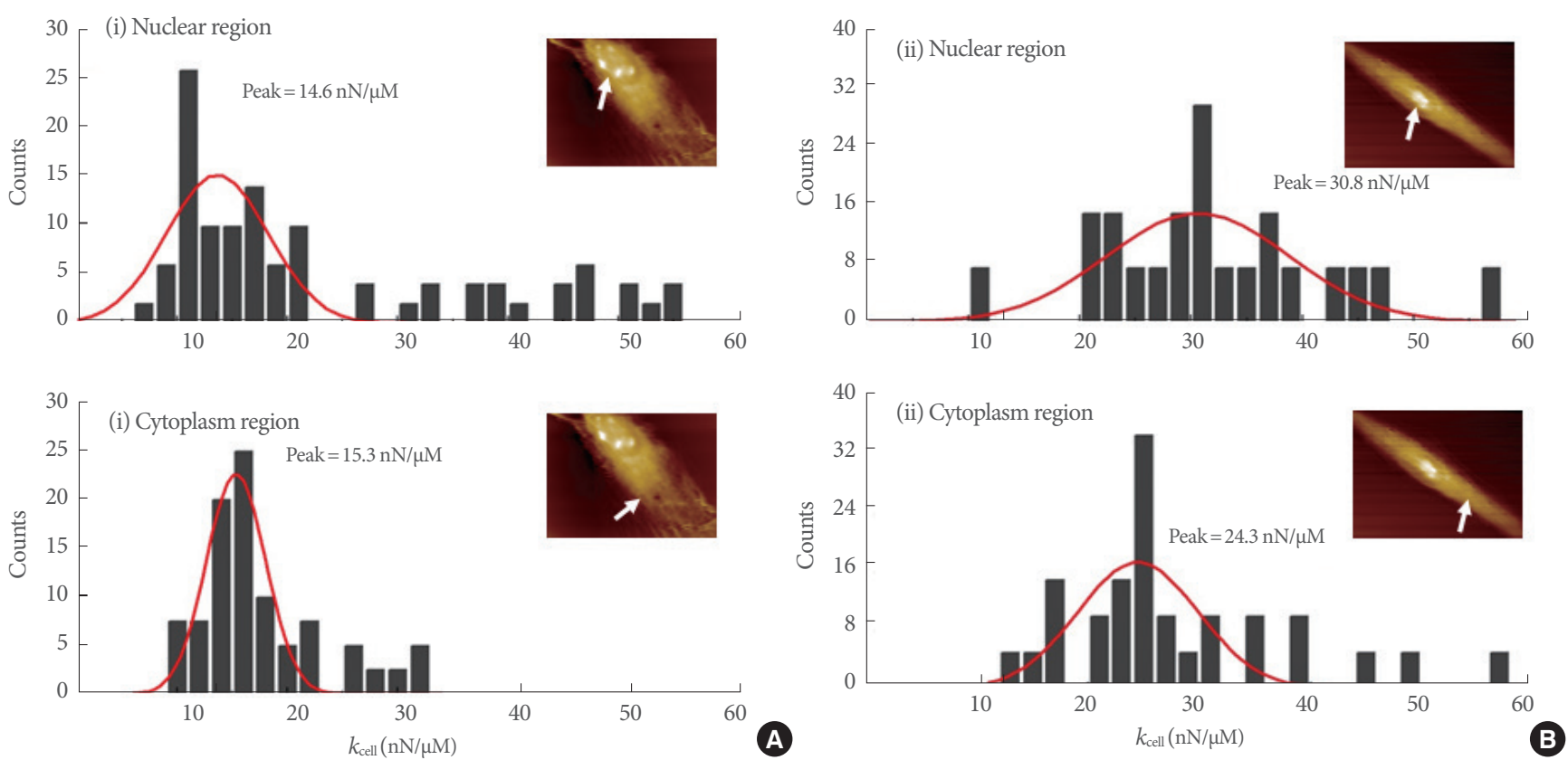

Supplementary Fig. 3. Distribution of stiffness in ADSCs (A) and UCSCs (B). Stiffness was measured separately in the nuclear and cytoplasm regions. Inset shows the atomic force microscopy image of ADSCs as an example of the region selected to measure the stiffness of a cell $\left(k_{\text {cell }}\right)$. The red lines indicate the Gaussian fit to the data. MSCs, mescenchymal stem cells; ADSCs, adipose-derived MSCs; UCSCs, umbilical cord-derived MSCs. 